Open Access

\title{
On the detection probability of the standard condition number detector in finite-dimensional cognitive radio context
}

\author{
Hussein Kobeissi ${ }^{1} 2^{*}$, Youssef Nasser $^{3}$, Amor Nafkha $^{1}$, Oussama Bazzi ${ }^{2}$ and Yves Louet ${ }^{1}$
}

\begin{abstract}
Standard condition number (SCN) detector is an efficient detector in multi-dimensional cognitive radio systems since no a priori knowledge is needed. The earlier studies usually assume a large number of dimensions and a large number of samples per dimension and use random matrix theory (RMT) to derive asymptotic distributions of the SCN metric. In practice, the number of dimensions may not be large enough for the SCN distribution to be well approximated by the asymptotic ones. In this context, the false alarm probability is considered in literature and formulas for 2D, 3D, and infinite-dimensional systems have been derived. However, the detection probability, which is of great importance in cognitive radio, has not been well discussed in literature. In this paper, we discuss, analytically, the detection probability of the SCN detector. Since the probability of detection is totally related to the SCN distribution, we derive new results on the joint ordered eigenvalues and SCN distributions for central semi-correlated Wishart matrices. These results are used to approximate the detection probability by the non-central/central approximation. We consider systems with three or more dimensions, and we give an approximated form of the detection probability. The analytical results of this paper on probability of detection along with those on probability of false alarm present a complete performance analysis and are validated through simulations. We show that the proposed analytical expressions provide high accuracy and that the SCN detector outperforms the well-known energy detector and the largest eigenvalue detector even with a small number of dimensions and low noise uncertainty environments.
\end{abstract}

Keywords: Cognitive radio, Joint distribution, Spectrum sensing, Standard condition number, Wishart matrix

\section{Introduction}

Cognitive radio (CR) is considered as a promising solution for the scarcity and inefficient use of the spectrum $[1,2]$. It allows the secondary (unlicensed) user (SU) to use the spectrum in an opportunistic way so that it does not cause harmful interference to the primary (licensed) user (PU). One possibility is to detect whether the PU is present or not to avoid any interference. Therefore, spectrum sensing has a key functionality in CR as it allows the $\mathrm{CR}$ to differentiate between the spectrum being used and the spectrum holes.

Several spectrum sensing techniques were proposed in the last decade [3]. Energy detector (ED) is among

\footnotetext{
*Correspondence: hussein.kobeissi.87@gmail.com

${ }_{1}^{1}$ SCEE/IETR, CentraleSupélec-Campus de Rennes, Rennes, France

${ }^{2}$ Department of Physics and Electronics, Faculty of Sciences 1, Lebanese University, Hadath, Beirut, Lebanon

Full list of author information is available at the end of the article
}

the most popular sensing techniques; it is simple, noncoherent, and needs no prior knowledge about the PU's signal. However, ED is very sensitive to noise variations and requires perfect knowledge of the noise power. Thus, the performance of ED suffers from a high degradation under low signal-to-noise ratio (SNR) and noise uncertainty conditions $[4,5]$.

Recently, eigenvalue-based detector (EBD) has been shown to overcome noise uncertainty challenges and performs adequately even in low SNR conditions. EBD is based on the eigenvalues of the received signal covariance matrix. In this context, the multi-dimensional aspect of a cognitive radio could be (i) multi-antenna CR $[6,7]$, (ii) cooperative scenario between several CR nodes [8], (iii) oversampling within the CR node [9], or any combination of them. If the entries within these dimensions are considered Gaussian, then the receiver's covariance matrix is known as a Wishart matrix in random matrix

\section{Springer Open}

(c) 2016 Kobeissi et al. Open Access This article is distributed under the terms of the Creative Commons Attribution 4.0 International License (http://creativecommons.org/licenses/by/4.0/), which permits unrestricted use, distribution, and reproduction in any medium, provided you give appropriate credit to the original author(s) and the source, provide a link to the Creative Commons license, and indicate if changes were made. 
theory (RMT) [10]. Results from RMT have been used to study the EBD algorithms which include the largest eigenvalue (LE) detector proposed in $[9,11]$, the scaled largest eigenvalue (SLE) detector [12, 13], and the standard condition number $(\mathrm{SCN})$ detector $[9,14-18]$. The LE is a semi-blind detector since it requires the knowledge of the noise power whereas the SLE and SCN are blind detectors that do not require this knowledge.

If we denote the maximum and the minimum eigenvalues of the receiver's covariance matrix by $\lambda_{\max }$ and $\lambda_{\min }$, respectively, then the standard condition number $(\mathrm{SCN})$ is defined as the ratio between $\lambda_{\max }$ and $\lambda_{\min }\left(\lambda_{\max } / \lambda_{\min }\right)$. In multiple-input multiple-output (MIMO) systems, the SCN has an effective connection with MIMO receiver performance in spatial multiplexing systems [19-21] and it indicates the multipath richness of the MIMO channel $[22,23]$. In CR systems, the SCN detector compares this ratio to a threshold to decide whether the PU exists or not. The performance of a detection algorithm is, usually, characterized by two types of probabilities: the false alarm probability $\left(P_{\mathrm{fa}}\right)$ and the detection probability $\left(P_{\mathrm{d}}\right)$. The latter depends on the decision threshold and are related to the statistics of the detector metric. Thus, the characteristics of the SCN are of great importance in both MIMO and CR systems.

The SCN metric was well studied in the recent years; for example, this metric was studied asymptotically in [14] and the threshold was presented according to MarchenkoPastur (MP) law [24]. If the size of the receiver's matrix is $K \times N$, then MP law proves that the largest $\left(\lambda_{\max }\right)$ and the smallest $\left(\lambda_{\min }\right)$ eigenvalues of the receiver's covariance matrix converge to constants as $(K, N) \rightarrow \infty$ with $c=K / N$ is a constant ratio [25]. These constants are simply determined by $c$ and the powers of the signal and the noise.

In [9], the authors improved the accuracy of the asymptotic statistical distribution of the SCN by using the TracyWidom (TW) distribution to model the largest eigenvalue (numerator of the SCN) [26] while maintaining the MP representation of the smallest one. TW distribution is a limiting distribution of the largest eigenvalue of a central Wishart matrix as $(K, N) \rightarrow \infty$ [27]. This approximation of the SCN, by TW distribution for the numerator and MP law for the denominator, results in an approximated relation between the decision threshold and the $P_{\mathrm{fa}}$.

This work was further extended in $[15,16]$ by using the Curtiss formula for the distribution of the ratio of random variables (i.e., SCN) [28] where both the largest and the smallest eigenvalues converge to Tracy-Widom distributions when $(K, N) \rightarrow \infty$ as shown in [26, 29]. Moreover, by the exploitation of the normal and TW distributions and using the Curtiss formula, the authors in [17] provided a form for the probability of missed detection $\left(P_{\mathrm{md}}=1-P_{\mathrm{d}}\right)$ for sufficiently large values of $K$ and
$N$. However, all these expressions include TW distribution and Curtiss formula that are hard to evaluate numerically online in addition to the considered asymptotic assumptions which are not necessarily practical.

By considering finite dimensions, the authors in [30] provided the exact generic form of the distribution of the $\mathrm{SCN}$ based on the joint distribution of the ordered eigenvalues of Wishart matrix. Consequently, [18] provides the exact forms of the $P_{\mathrm{fa}}$ of the $\mathrm{SCN}$ detector for CR with two dimensions and [31] provides $P_{\mathrm{fa}}$ form for three dimensions. In addition, the $P_{\mathrm{d}}$ for two-dimensional systems was approximated using the non-central/central approximation and provided in [18] following the results in [30]. When $K \geq 3$ (for $K$-dimensional system), the form of the $P_{\mathrm{d}}$ could be derived using the results in [30] as long as the non-centrality matrix $\Omega_{K}$ is full rank. Moreover, the non-central/central approximation is used to approximate the distribution of the non-central uncorrelated Wishart matrix by the distribution of the central semi-correlated Wishart matrix. However, using this method to approximate the distribution of the $\mathrm{SCN}$ requires normalization and the behavior of this approximation could be affected especially at high SNR.

In this paper, the non-centrality matrix $\left(\Omega_{K}\right)$ is not a full-rank matrix as considered in previous studies $[18,30]$. This case includes, for example, but is not limited to, a single PU transmitting orthogonal frequency-division multiplexing (OFDM) signal. Accordingly, results derived in literature may not be applicable since they are derived for the full rank case. Thus, by using the non-central/central approximation, we provide the new form of the $P_{\mathrm{d}}$ of the SCN metric for general finite $K$-dimensional systems and we give the form in the particular case when $K=3$. Also, we study the impact of the system parameters $(K, N$, and SNR) on the approximation accuracy and on the detection probability. The main contribution of this paper could be summarized as follows:

- Using the non-central/central approximation, the eigenvalues of the correlation matrix of the central semi-correlated Wishart matrix may coincide. For this purpose, we generalize the joint distribution of the ordered eigenvalues of the central semi-correlated Wishart matrix. In our derivations, the eigenvalues of the correlation matrix can be equal or distinct.

- The authors in [30] consider the case where all the eigenvalues of the correlation matrix are distinct. In this paper, we show that the generic form of the cumulative distribution function (CDF) of the SCN given in [30] is still valid if the eigenvalues are not distinct. However, the form of the parameters differ where we give the new forms.

- We derive the analytical forms of the CDF and the probability density function (PDF) of the $\mathrm{SCN}$ of 
three-dimensional systems when $\Omega_{K}$ is not a full-rank matrix.

- For the sake of completeness, we also derive the CDF and the PDF of the SCN of three-dimensional systems when $\Omega_{K}$ is a full-rank matrix. Thus, the results of this paper combined with results in [31] form a complete closed-form system for the three-dimensional cognitive radio.

- Since the previous studies consider the noncentral/central approximation in low SNR regions [18], we study the impact of the system parameters on the accuracy of the non-central/central approximation. This impact is given in terms of $P_{\mathrm{d}}$ as it is an important target in the general CR context.

The rest of this paper is organized as follows. In Section 2, the system model is introduced and discussed. In Section 3, the non-central/central approximation is recalled and analyzed. In Section 4, we give the joint ordered eigenvalue distribution with a generalization to equal eigenvalues of the correlation matrix. In Section 5, we emphasize on the general form of the CDF of the SCN for the central semi-correlated Wishart matrix when $L$ eigenvalues of the correlation matrix are equal. The exact CDF and PDF of this SCN for three-dimensional systems are derived as well. Simulation results and approximation accuracy are discussed in Section 6, and we conclude in Section 7.

Notations: Vectors and matrices are represented, respectively, by lowercase and uppercase boldface. The symbols $|$.$| and \operatorname{tr}($.$) represent, respectively, the determi-$ nant and trace of a matrix while $(.)^{1 / 2},(.)^{\mathrm{T}}$, and $(.)^{\dagger}$ are the square root, transpose, and Hermitian symbols, respectively. $I_{n}$ is the $n \times n$ identity matrix. Symbol $\|\cdot\|^{2}$ stands for the norm, $E[$.$] stands for the expected value, and \sim$ stands for distributed as.

\section{System model}

We consider a $K$-dimensional $C R$ system, and we aim to detect the presence/absence of a single PU during a sensing period which corresponds to $N$ samples. The $K$ dimensions might be $K$ collaborating SUs, $K$ antennas, an oversampling by a factor of $K$, or any combination of them. For this detection problem, there are two hypotheses: $\mathcal{H}_{0}$ corresponds to the absence of PU transmission (i.e., spectrum hole) and $\mathcal{H}_{1}$ corresponds to the presence of the PU transmission (i.e., spectrum being used). The received vector, at instant $n$, under each hypothesis is given by

$$
\begin{aligned}
& \mathcal{H}_{0}: \boldsymbol{y}(n)=\boldsymbol{\eta}(n), \\
& \mathcal{H}_{1}: \boldsymbol{y}(n)=\boldsymbol{h}(n) s(n)+\boldsymbol{\eta}(n),
\end{aligned}
$$

where $\boldsymbol{y}(n)=\left[y_{1}(n), \cdots, y_{K}(n)\right]^{\mathrm{T}}$ is the observed $K \times 1$ complex samples across all the dimensions at instant $n$. $\boldsymbol{\eta}(n)$ is a $K \times 1$ complex circular white Gaussian noise. $\boldsymbol{h}(n)$ is a $K \times 1$ complex vector that represents the channel coefficients between the PU and each of the CR dimensions, and $s(n)$ stands for the primary signal sample at instant $n$.

After collecting $N$ samples from each dimension, the received signal matrix $\boldsymbol{Y}$ is written as follows:

$$
\boldsymbol{Y}=\left(\begin{array}{cccc}
y_{1}(1) & y_{1}(2) & \cdots & y_{1}(N) \\
y_{2}(1) & y_{2}(2) & \cdots & y_{2}(N) \\
\vdots & \vdots & \ddots & \vdots \\
y_{K}(1) & y_{K}(2) & \cdots & y_{K}(N)
\end{array}\right)
$$

Without loss of generality, we suppose that $K \leq N$ and we define the received sample covariance matrix as $W=Y Y^{\dagger}$.

Under $\mathcal{H}_{0}$, the input of the matrix $Y$ is a complex circular white Gaussian noise with zero mean and unknown variance $\sigma_{\eta}^{2}$, then $\boldsymbol{W}$ is well known as a central uncorrelated complex Wishart matrix and is denoted by $W \sim$ $\mathcal{C W}_{K}\left(N, \sigma_{\eta}^{2} \mathbf{I}_{K}\right)$ where $K$ is the size of the matrix, $N$ is the number of degrees of freedom (DoF), and $\sigma_{\eta}^{2} \mathbf{I}_{K}$ is the correlation matrix.

Under $\mathcal{H}_{1}$, we suppose that the channel remains constant during the sensing time and we have a single PU whose signal is drawn independently from Gaussian process for every sample. Consequently, $W$ follows a noncentral uncorrelated complex Wishart distribution which is denoted as $W \sim \mathcal{C} \mathcal{W}_{K}\left(N, \sigma_{\eta}^{2} \mathbf{I}_{K}, \Omega_{K}\right)$, where $\Omega_{K}$ is a rank 1 non-centrality matrix [32].

Let us denote by $\lambda_{1} \geq \lambda_{2} \geq \cdots \geq \lambda_{K}>0$ the eigenvalues of $W$, then the SCN metric is given by

$$
\mathrm{SCN}=\frac{\lambda_{1}}{\lambda_{K}}
$$

Denoting by $t$ the decision threshold, then the detection probability $\left(P_{\mathrm{d}}\right)$, defined as the probability of correctly detecting the presence of $\mathrm{PU}$, and the false alarm probability $\left(P_{\mathrm{fa}}\right)$, defined as the probability of detecting the presence of PU while it does not exist, are, respectively, given by (5) and (6) and shown in Fig. 1.

$$
\begin{aligned}
& P_{\mathrm{d}}=P\left(\mathrm{SCN} \geq t / \mathcal{H}_{1}\right) \\
& P_{\mathrm{fa}}=P\left(\mathrm{SCN} \geq t / \mathcal{H}_{0}\right) .
\end{aligned}
$$

These probabilities depend on the threshold $(t)$ being used. However, if the expressions of the $P_{\mathrm{fa}}$ and $P_{\mathrm{d}}$ are previously known, then a threshold could be set according to a required error constraint. Then, it is clear that these probabilities depend on the distribution of the SCN metric. If we denote the $\mathrm{CDF}$ and $\mathrm{PDF}$ of $\mathrm{SCN}$, respectively, by $F_{i}($.$) and f_{i}($.$) with index i \in\{0,1\}$ indicating the considered hypothesis, then we can write 


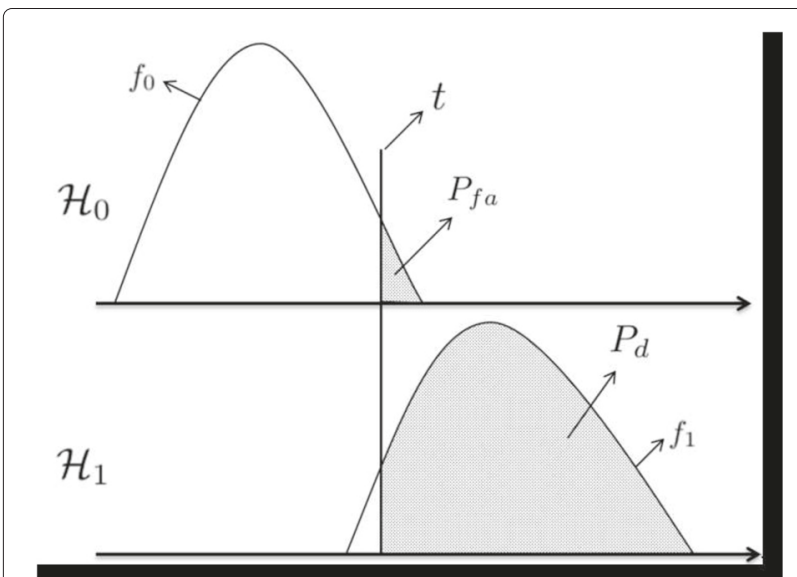

Fig. 1 Detection probability $\left(P_{\mathrm{d}}\right)$ and false alarm probability $\left(P_{\mathrm{fa}}\right)$ as a function of the decision threshold $(t)$ under both hypotheses

$$
\begin{aligned}
& P_{\mathrm{fa}}=1-F_{0}(t) \\
& P_{\mathrm{d}}=1-F_{1}(t)
\end{aligned}
$$

The exact general form of the distribution of SCN under $\mathcal{H}_{0}\left(F_{0}(t)\right)$ has been derived in [30]. Two particular cases have been considered in $[18,31]$ when $K=2$ and 3 , respectively. Asymptotically, this distribution could be found in $[9,14,15]$. Under $\mathcal{H}_{1}$, an asymptotic distribution of the SCN, $F_{1}(t)$, could be found in [17]. In the finite case, the exact generic form of $F_{1}(t)$ is derived in [30]; however, further numerical evaluation would either require Nuttall-Q function which could be replaced by Marcum Q-function and a finite weighted sum of Bessel functions [33] or by hypergeometric functions that could be expanded to an infinite sum (see, for example, [34] for $K=2$ and $N=2$ ). Thus, and since both solutions are difficult to manipulate, ${ }^{1}$ a third solution is to use the non-central/central approximation that approximates the distribution of the non-central uncorrelated Wishart matrix by the distribution of the central semi-correlated Wishart matrix. This approximation is presented in the next section. The exact general form of the distribution of the SCN of central semi-correlated Wishart matrix is provided by [30] and is used to approximate $F_{1}(t)$ for $K=2$ in $[18,30]$.

\section{Non-central/central approximation and parameter analysis}

The non-central/central approximation is recalled in Lemma 1.

Lemma 1 ([35]). The complex non-central Wishart matrix $W \sim \mathcal{C W}_{K}\left(N, \kappa^{2} \mathbf{I}_{K}, \Omega_{K}\right)$ and the complex semicorrelated Wishart matrix $W \sim \mathcal{C W}_{K}\left(N, \widehat{\Sigma}_{K}\right)$ with effective correlation matrix $\widehat{\Sigma}_{K}=\kappa^{2} I_{K}+\Omega_{K} / N$ have the same first-and second-order moments differing by ${ }^{\Omega_{K}} /{ }_{N}$.
Using Lemma 1, the distribution of the SCN under $\mathcal{H}_{1}$ hypothesis could hence be approximated by the distribution of the SCN of complex semi-correlated Wishart matrix with a proper correlation matrix. It is worth noting that this approximation for the $\mathrm{SCN}$ distribution also requires a normalization by $\kappa^{2}\left(\mathbf{W}_{n}=\kappa^{-2} \mathbf{W}\right)$ which gives the same SCN as W. Therefore, following (8), the detection probability is, accordingly, approximated.

Under hypothesis $\mathcal{H}_{1}$ and following our assumptions, the non-centrality matrix $-\Omega_{K}$ is a rank 1 matrix and the only non-zero eigenvalue of $\Omega_{K}$ is denoted by $\omega_{1}$. Consequently, and after applying normalization of Lemma 1 , the effective correlation matrix, $\widehat{\Sigma}_{K}$, has coincidence in its eigenvalues. Let $\sigma=\left[\sigma_{1}, \sigma_{2}, \cdots, \sigma_{K}\right]^{\mathrm{T}}$ be the vector of ordered eigenvalues of $\widehat{\Sigma}_{K}=I_{K}+\Omega_{K} / N$, then all but one eigenvalue of $\widehat{\Sigma}_{K}$ are still equal to 1 (eigenvalues of $I_{K}$ ) while $\sigma_{1}$ is given by

$$
\sigma_{1}=1+\frac{\omega_{1}}{N}
$$

The average SNR, under $\mathcal{H}_{1}$, is defined by

$$
\rho=\frac{\sigma_{s}^{2} \sigma_{h}^{2}}{\sigma_{\eta}^{2}}
$$

where the PU signal power, $\sigma_{s}^{2}$, could be estimated by ${ }^{2}$ $\left(\|\boldsymbol{s}\|^{2} / N\right)$, and the channel power $\sigma_{h}^{2}=\left(\|\boldsymbol{h}\|^{2} / K\right)$. The non-centrality matrix is given by

$$
\Omega_{K}=\Sigma_{K}^{-1} \boldsymbol{M} \boldsymbol{M}^{\dagger}=\frac{1}{\sigma_{\eta}^{2}}\|\boldsymbol{s}\|^{2} \boldsymbol{h} \boldsymbol{h}^{\dagger},
$$

where $\Sigma_{K}$ is the covariance matrix of $Y$, defined as $\Sigma_{K}=E\left[(\boldsymbol{Y}-\boldsymbol{M})(\boldsymbol{Y}-\boldsymbol{M})^{\dagger}\right]=\sigma_{\eta}^{2} I_{K}$, and $\boldsymbol{M}$ is the mean of $\boldsymbol{Y}$ defined as $\boldsymbol{M}=E[\boldsymbol{Y}]=\boldsymbol{h s}^{\mathrm{T}}$ with $\boldsymbol{h}=\left[h_{1} h_{2} \cdots h_{K}\right]^{\mathrm{T}}$ and $s=[s(1) s(2) \cdots s(N)]^{\mathrm{T}}$. It is worth mentioning that (11) will not be changed after normalization.

As a result, and by using the property that the trace of a matrix equals the sum of its eigenvalues, then $\omega_{1}$ could be written as

$$
\begin{aligned}
\omega_{1} & =\operatorname{tr}\left(\Omega_{K}\right)=\frac{1}{\sigma_{\eta}^{2}}\|\boldsymbol{s}\|^{2} \operatorname{tr}\left(\boldsymbol{h} \boldsymbol{h}^{\dagger}\right)=\frac{1}{\sigma_{\eta}^{2}}\|\boldsymbol{s}\|^{2}\|\boldsymbol{h}\|^{2} \\
& =N K \rho .
\end{aligned}
$$

Thus, $\Omega_{K}$ is not full rank and the eigenvalues of $\widehat{\Sigma}_{K}$ are not distinct. It is worth noting that for the exact form of $P_{\mathrm{d}}$, it would be useful to refer to the joint distribution of the ordered eigenvalues of non-central uncorrelated Wishart matrix with arbitrary non-centrality matrix rank derived in [36, App. I] and consider rank 1 case. However, as discussed before, it is hard to derive a tractable form for the SCN distribution since it requires Nuttall-Q functions or hypergiometric functions. Therefore, by considering the non-central/central approximation, results based on assuming all correlation eigenvalues distinct could not be 
used and new results must be derived. Accordingly, an approximated and simpler form for the $P_{d}$ will be derived.

\section{Joint ordered eigenvalues distribution generalization}

As discussed in the previous section, all but one of the eigenvalues of the correlation matrix are equal. In this section, and since the distribution of the SCN metric is derived using the joint distribution of the eigenvalues of the Wishart matrix, we generalize the joint distribution of the ordered eigenvalues of the central semi-correlated Wishart matrix. This new form of the joint distribution covers any number $(L)$ of equal eigenvalues positioned anywhere in vector $\sigma$ and is given in Theorem 1 below.

In addition, Theorem 1 is also useful in MIMO systems as it could be used to derive marginal and joint distributions in correlated Rayleigh channels when the correlation eigenvalues may coincide. Moreover, these derivations could, also, approximate that of the Rician channels when the mean channel matrix is of rank 1; this could be satisfied when all channel vectors have the same mean value or in some other practical scenarios where the line-of-sight (LOS) component of the channel matrix approaches the scaled all-ones matrix [37].

Theorem 1 (Central semi-correlated Wishart when $\boldsymbol{\Sigma}_{K}$ is full rank with $L$ equal eigenvalues). Let $W$ be a central semi-correlated Wishart matrix $\mathbb{W} \sim \mathcal{C W}_{K}\left(N, \Sigma_{K}\right)$ and denote by $\sigma_{1}>\sigma_{2}>\cdots>\sigma_{p}=\cdots=\sigma_{q}>\cdots>\sigma_{K}>0$ the ordered eigenvalues of $\boldsymbol{\Sigma}_{K}$ assumed full rank with $L$ equal eigenvalues (from $p$ to $q$ with $q=p+L-1$ ), then the joint PDF of $\lambda$ could be written as follows:

$$
f(\lambda)=C|\boldsymbol{\Phi}(\lambda)| \times|\boldsymbol{\Psi}(\lambda)| \prod_{i=1}^{K} \xi\left(\lambda_{i}\right)
$$

\section{with the associated parameters defined as}

- $\boldsymbol{\Phi}(\lambda)$ is a $K \times K$ Vandermonde matrix of entries $\lambda_{j}^{i-1}$.

- $\boldsymbol{\Psi}(\lambda)$ is a $K \times K$ matrix of the form $\mathbf{E}(\lambda, \sigma)$ given, for all $1 \leq j \leq K$, by

$$
\{\mathbf{E}(\lambda, \boldsymbol{\sigma})\}_{i, j}=\left\{\begin{array}{cc}
\frac{\partial^{q-i}\left(e^{-\frac{\lambda_{j}}{\sigma_{p}}}\right)}{\partial \sigma_{p}^{q-i}} & p \leq i \leq q \\
e^{-\frac{\lambda_{j}}{\sigma_{i}}} & \text { otherwise }
\end{array}\right.
$$

- $\xi\left(\lambda_{i}\right)$ is defined as $\xi\left(\lambda_{i}\right)=\lambda_{i}^{N-K}$.

- The normalization constant is given by

$$
C=\frac{\prod_{i<j}^{K} \sigma_{i} \sigma_{j}}{\prod_{i=1}^{K}(N-i) ! \sigma_{i}^{N} \prod_{\substack{i<j \\ \sigma_{i} \neq \sigma_{j}}}^{K}\left(\sigma_{j}-\sigma_{i}\right) \Gamma_{L}(L)},
$$

where $\Gamma_{s}(l)=\prod_{i=1}^{s}(l-i)$ ! and

$$
\frac{\partial^{n}\left(e^{-\frac{\lambda_{j}}{\sigma_{p}}}\right)}{\partial \sigma_{p}^{n}}=\left\{\begin{array}{cl}
e^{-\frac{\lambda_{j}}{\sigma_{p}}} \sum_{k=1}^{n} \frac{(-1)^{k+n} \cdot \mathcal{L}(n, k) \cdot \lambda_{j}^{k}}{\sigma_{p}^{n+k}} & \text { if } n>0 \\
e^{-\frac{\lambda_{j}}{\sigma_{p}}} & \text { if } n=0
\end{array}\right.
$$

with $\mathcal{L}(n, k)$ is the Lah number defined by

$$
\mathcal{L}(n, k)=\left(\begin{array}{l}
n \\
k
\end{array}\right)\left(\begin{array}{l}
n-1 \\
k-1
\end{array}\right)(n-k) !
$$

Proof. See Appendix 1.

It can be easily shown that by taking $L=1$, the parameters in Theorem 1 will be equivalent to the case of all distinct eigenvalues in the literature [30, Table I].

\section{SCN distribution}

In this section, we will derive the general form of the CDF of the SCN of central semi-correlated Wishart matrix for arbitrary $K$ and $N$. Then, we emphasize by considering the three-dimensional case $(K=3)$. Given $(8)$ and the results of this section, $P_{\mathrm{d}}$ can then be directly computed.

\subsection{The general form}

The CDF, $F(x)$, with $x \geq 1$, of the SCN of the central semi-correlated Wishart matrix $\mathbf{W} \sim \mathcal{C W}_{K}\left(N, \Sigma_{K}\right)$ with distinct correlation eigenvalues, denoted by $\sigma_{1}>\sigma_{2}>$ $\cdots>\sigma_{K}>0$, is given by [30]

$$
F(x)=C \sum_{l=1}^{K} \int_{0}^{\infty}\left|\left[\begin{array}{cc}
\int_{\lambda_{K}}^{x \lambda_{K}} \phi_{i}(u) \psi_{j}(u) \xi(u) d u, & i \neq l \\
\phi_{i}\left(\lambda_{K}\right) \psi_{j}\left(\lambda_{K}\right) \xi\left(\lambda_{K}\right), & i=l
\end{array}\right]\right| d \lambda_{K},
$$

where $\phi_{i}\left(\lambda_{j}\right)=[\boldsymbol{\Phi}(\lambda)]_{i, j}$ and $\psi_{i}\left(\lambda_{j}\right)=[\boldsymbol{\Psi}(\lambda)]_{i, j}$ with $\boldsymbol{\Phi}(\lambda)$, $\boldsymbol{\Psi}(\lambda), \xi\left(\lambda_{i}\right)$, and $C$ are defined in [30, Table I].

If we have $L$ equal correlation eigenvalues (say from $p$ to $q$ with $q=p+L-1)$, then (18) is still valid by considering the parameters $\boldsymbol{\Phi}(\lambda), \boldsymbol{\Psi}(\lambda), \xi\left(\lambda_{i}\right)$, and $C$ that are defined in Theorem 1 . This could be easily shown by comparing the joint distribution parameters in Theorem 1 and Table I of [30] and noting that they have the same general form used in deriving the SCN CDF in (18).

It is important to note that all the integrals inside the determinant in (18), $I_{i, j}=\int_{\lambda_{K}}^{x \lambda_{K}} \phi_{i}(u) \psi_{j}(u) \xi(u) d u$, still admit a closed-form solution given, directly after integrating using [38, Eq. (3.351.1)], by 


$$
I_{i, j}=\left\{\begin{array}{ll}
{\left[\gamma\left(l, \frac{x \lambda_{K}}{\sigma_{j}}\right)-\gamma\left(l, \frac{\lambda_{K}}{\sigma_{j}}\right)\right] \cdot \sigma_{j}^{l}} & i<p \text { or } i \geq q \\
\sum_{k=1}^{q-j}\left[(-1)^{k+q-j} \mathcal{L}(q-j, k) \cdot\left[\gamma\left(l+k, \frac{x \lambda_{K}}{\sigma_{p}}\right)-\gamma\left(l+k, \frac{\lambda_{K}}{\sigma_{p}}\right)\right]\right] \sigma_{p}^{l+j-q} p \leq i<q
\end{array},\right.
$$

where $\gamma(.,$.$) is the lower incomplete gamma function$ [38, Eq. (8.350.1)], the constant $l=N-K+i$, and $1 \leq i \leq K$.

\subsection{SCN distribution for three-dimensional systems} In the previous sections, we have considered an arbitrary number $(L)$ of equal eigenvalues positioned anywhere in vector $\sigma$. Considering our assumptions that $\Omega_{K}$ is a rank 1 matrix reveals, from Lemma 1 , that the eigenvalues of the effective correlation matrix of the central semi-correlated Wishart matrix has $(K-1)$-equal eigenvalues such that $\sigma_{1}>\sigma_{2}=\cdots=\sigma_{K}>0$.

Now, if we consider the case $K=3$, then $\widehat{\Sigma}_{K}$ has two equal eigenvalues $\left(\sigma_{1}>\sigma_{2}=\sigma_{3}>0\right)$. In this case, the $\mathrm{CDF}$ and PDF of the SCN are given in Theorem 2 and Corollary 1, respectively.

Theorem 2 (CDF of SCN of central semi-correlated Wishart matrix when $\Sigma_{3}$ has two equal eigenvalues). The CDF of the SCN of a $3 \times 3$ central semi-correlated Wishart matrix $\mathbf{W} \sim \mathcal{C W}_{K}\left(N, \Sigma_{3}\right)$ whose $\Sigma_{3}$ has two equal eigenvalues $\left(\sigma_{1}>\sigma_{2}=\sigma_{3}\right)$ is given by

$$
F_{1}(x)=C_{s c}\left[R_{1}(N, x)-G_{1}(N, x)\right],
$$

where $R_{1}(N, x)$ is defined in (21), $G_{1}(N, x)$ in (22), $S_{1}(r, s, t, \mu, v, x)$ in (23), $\Delta_{1}(r, s, t, \mu, v, \varepsilon, x)$ in (24), and $C_{s c}$ in (25).

$$
\begin{aligned}
R_{i}(N, x)= & S_{i}\left(N-3, N, N-2, \sigma_{1}^{-1}, \sigma_{2}^{-1}, x\right) \\
& +S_{i}\left(N-2, N-2, N-1, \sigma_{1}^{-1}, \sigma_{2}^{-1}, x\right) \\
& +S_{i}\left(N-1, N-1, N-3, \sigma_{1}^{-1}, \sigma_{2}^{-1}, x\right), i=1,2
\end{aligned}
$$

$$
\begin{aligned}
G_{i}(N, x)= & \Delta_{i}\left(N-2, N-1, N-2, \sigma_{2}^{-1}, \sigma_{2}^{-1}, \sigma_{1}^{-1}, x\right) \\
& +\Delta_{i}\left(N-2, N, N-3, \sigma_{2}^{-1}, \sigma_{2}^{-1}, \sigma_{1}^{-1}, x\right) \\
& +\Delta_{i}\left(N-3, N-1, N-1, \sigma_{2}^{-1}, \sigma_{2}^{-1}, \sigma_{1}^{-1}, x\right), i=1,2
\end{aligned}
$$

$$
\begin{aligned}
S_{i}(r, s, t, \mu, \nu, x)= & \Delta_{i}(r, s, t, \nu, \nu, \mu, x)+\Delta_{i}(t, r, s, \nu, \mu, \nu, x) \\
& -\Delta_{i}(r, t, s, \nu, \mu, \nu, x)-\Delta_{i}(r, s, t, v, \mu, \nu, x) \\
& +\Delta_{i}(t, s, r, v, \mu, \nu, x), \quad i=1,2
\end{aligned}
$$

$$
\begin{aligned}
& \Delta_{1}(r, s, t, \mu, v, \varepsilon, x)=-\mu^{2} \Delta_{3}(r, s, t, v, \varepsilon, \mu, x) \\
& C_{s c}=\frac{\left(\sigma_{2}-\sigma_{1}\right)^{-2}}{(N-1) !(N-2) !(N-3) ! \sigma_{1}^{N-2} \sigma_{2}^{2(N-2)}}
\end{aligned}
$$

with $\Delta_{3}$ defined in (35) in Appendix 2, Theorem 3.

Proof. The proof could be summarized as follows:

1. Considering (18) and setting $K=3$.

2. Substituting the parameters from Theorem 1.

3. Expanding the summation and using (19).

4. Expanding the determinant and integrating using Eqs. (3.351.1) and (3.351.3) of [38].

Then, the result comes after simplification.

Corollary 1. The PDF of the SCN of a $3 \times 3$ central semicorrelated Wishart matrix $\mathbf{W} \sim \mathcal{C W}_{K}\left(N, \Sigma_{3}\right)$ whose $\Sigma_{3}$ has two equal eigenvalues $\left(\sigma_{1}>\sigma_{2}=\sigma_{3}\right)$ is given by

$$
f_{1}(x)=C_{s c} \cdot\left[R_{2}(N, x)-G_{2}(N, x)\right],
$$

where $R_{2}(N, x)$ is defined in (21), $G_{2}(N, x)$ in (22), $S_{2}(r, s, t, \mu, v, x)$ in (23), $C_{s c}$ in (25), and $\Delta_{2}(r, s, t, \mu, v, \varepsilon, x)$ in (27) below.

$$
\Delta_{2}(r, s, t, \mu, v, \varepsilon, x)=-\mu^{2} \Delta_{4}(r, s, t, v, \varepsilon, \mu, x)
$$

with $\Delta_{4}$ defined in (38) in Appendix 2, Corollary 2.

Proof. Obtained by differentiating Eq. (20).

For the sake of completeness, the reader could find, in Appendix 2, the CDF and PDF of the SCN of the central semi-correlated Wishart matrix when all the correlation eigenvalues are distinct.

\section{Simulation and discussion}

In this section, we discuss the analytical results through Monte Carlo simulations. We, firstly, validate the theoretical analysis presented in Sections 4 and 5. Given the noncentral/central approximation in Lemma 1, we study the approximation accuracy as well as the impact of the SNR on this approximation. Since the probability of detection is the main target, we study the effect of the approximation accuracy and the impact of system parameters on $P_{\mathrm{d}}$. Finally, we show that the SCN detector outperforms the $\mathrm{ED}$ and the LE detector if noise power is not perfectly known. 
For this purpose, we, first, generate $10^{5}$ central semicorrelated Wishart matrices. This type of matrices can be easily generated under $\mathcal{H}_{0}$ by considering a certain correlation between different dimensions of the $C R$ system. The received matrix, under $\mathcal{H}_{0}$ hypothesis, is then written as

$$
\mathbf{Y}=\Sigma_{K}^{1 / 2} \mathbf{G}
$$

where $\boldsymbol{G}$ is the Gaussian noise matrix and $\boldsymbol{\Sigma}_{K}$ is the correlation matrix.

Figures 2 and 3 validate the analytical form of the distribution of the SCN of the central semi-correlated Wishart matrix derived in Section 5. A three-dimensional system is considered, and the receiver matrix is given in (28). The correlation matrix, $\Sigma_{K}$, is modeled according to the normalization of Lemma 1 ; thus, $\sigma_{1}=1+K \rho$ and $\sigma_{2}=\sigma_{3}=1$. Figure 2 shows the impact of varying the number of received samples $(N)$ while the SNR $(\rho)$ variation is considered in Fig. 3. Results show a perfect match between the empirical SCN distribution of the central semi-correlated Wishart matrix and the analytical form in Section 5. Moreover, these figures show a validation of the joint distribution given in Theorem 1 .

Central/non-central approximation accuracy is shown in Fig. 4. In this case, we consider a BPSK modulated signal and a complex Gaussian noise with unit variance $\left(\sigma_{\eta}^{2}=1\right)$. Since we have considered a flat fading channel, we suppose that the signal will experience a unity fading magnitude. Figure 4 gives the empirical distribution of the SCN of non-central uncorrelated Wishart matrices (under $\mathcal{H}_{1}$ hypothesis) and its corresponding normalized analytical approximation using Lemma 1 for different SNR values $(\rho)$. Results show a perfect match between the empirical results and the analytical approximation at low SNR values, however, approximation accuracy degrades as SNR increases $(\rho>-2 \mathrm{~dB})$.

Figures 5 and 6 present the impact of the system parameters $(K, N$, and $\rho)$ on the approximation in terms of

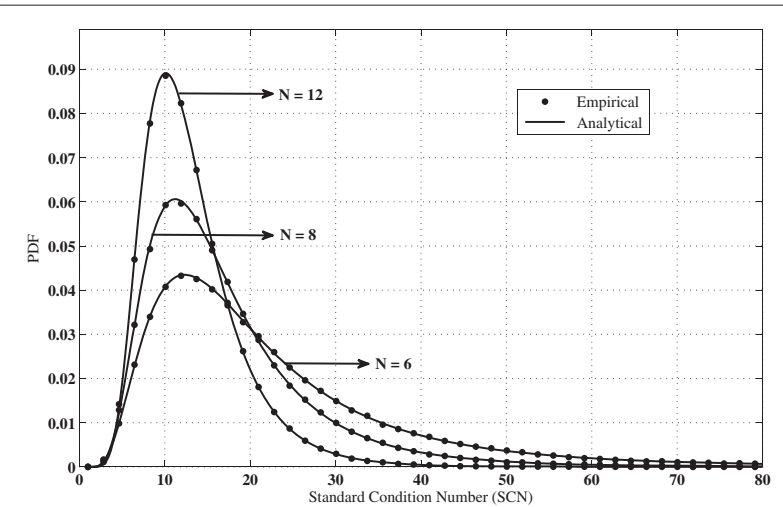

Fig. 2 Empirical and analytical probability density functions of the $\mathrm{SCN}$ of the central semi-correlated Wishart matrices as $N$ varies

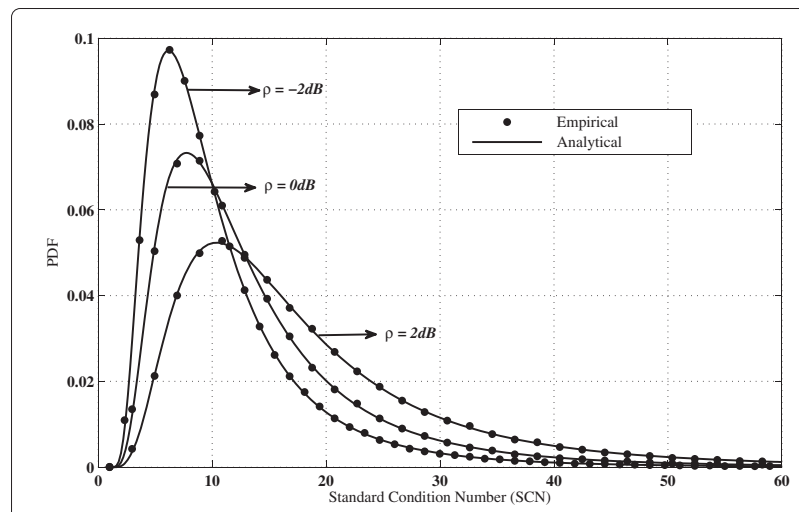

Fig. 3 Empirical and analytical probability density function of the SCN of the central semi-correlated Wishart matrices as $\rho$ varies

the performance of the $\mathrm{SCN}$ detector for a preset target $P_{\mathrm{fa}}$. Figure 5 shows the $P_{\mathrm{d}}$ of the SCN detector of threedimensional $C R$ as a function of SNR for different number of samples $(N=\{10,30,50,100\})$ while in Fig. 6, we vary $K$ $(K=\{2,3\})$ with the false alarm being set to to $P_{\mathrm{fa}}=0.1$. Both figures show perfect accuracy for low SNR values $(\rho<-2 \mathrm{~dB})$; however, as SNR increases the approximation starts to show dissimilarity with the empirical results until both probabilities reach 1 (both distributions, the empirical and the analytical, are totally to the right of the considered threshold).

Figure 5 shows that the dissimilarity between the curves decreases as $N$ increases and becomes very close to zero at $N=100$. This is related to the threshold selection criteria. In CR, the threshold is-usually-selected according to a target $P_{\mathrm{fa}}$ (i.e., the distribution of the $\mathrm{SCN}$ under $\mathcal{H}_{0}$ as shown in Fig. 1). As $N$ increases, the detection probability is improved as the part of the distribution under $\mathcal{H}_{1}$ to the right of the threshold increases. This reveals that,

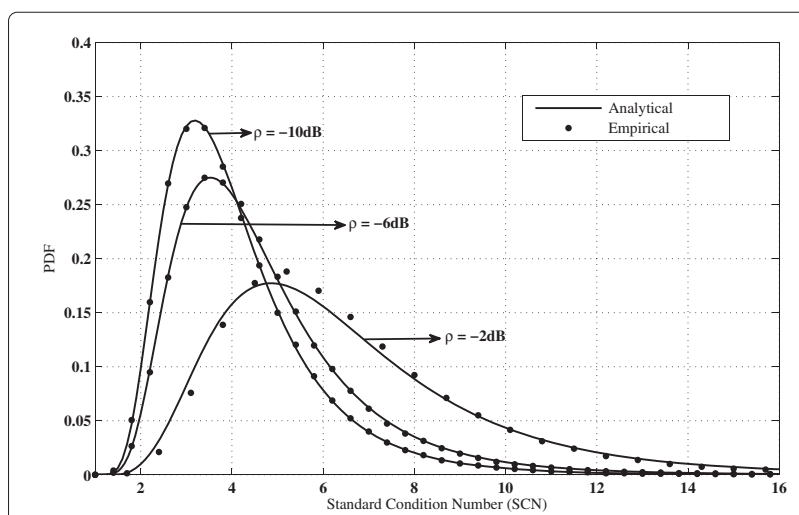

Fig. 4 Empirical probability density function of the SCN of non-centra uncorrelated Wishart matrices and the corresponding analytical probability density function of central semi-correlated Wishart matrices using normalized non-central/central approximation 


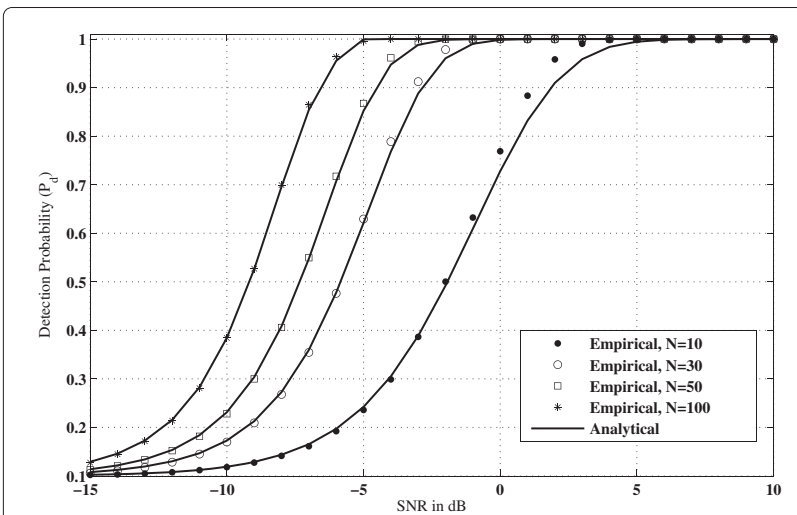

Fig. 5 Empirical detection probability of the SCN metric and its corresponding analytical detection probability using normalized non-central/central approximation as a function of SNR for $K=3$ and $P_{\mathrm{fa}}=0.1$ in the sense of detection performance, the normalized non-central/central approximation could be considered as a good fit for the empirical distribution of the $\mathrm{SCN}$ for sufficiently large values of $N$. Indeed, both distributions perfectly match at low SNR values and exceed the threshold for higher SNR values where the approximation accuracy degrades. Moreover, the same result could be deduced from Fig. 6 where the performance of the detector increases as $K$ increases.

To stand on the previous results and to validate the approximation accuracy obtained at low SNR for different values of $N$ and $K$, we present in Fig. 7 the $P_{\mathrm{d}}$ of SCN metric for different $N$ and $K$ with SNR fixed at $\rho=-10 \mathrm{~dB}$ and $P_{\mathrm{fa}}=0.1$. The figure shows a perfect match between the empirical and the analytical results for all values of $K$ and $N$. As it could be seen from the figure, the distribution under $\mathcal{H}_{1}$ hypothesis partially exceeds the threshold

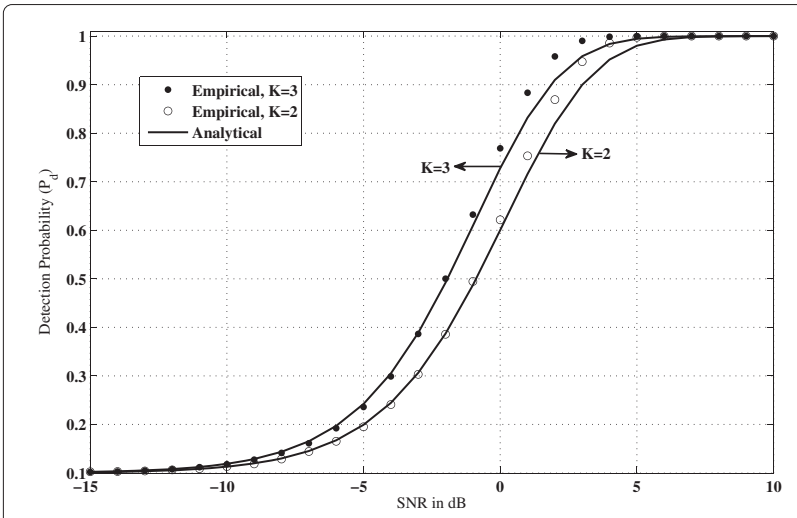

Fig. 6 Empirical detection probability of the SCN metric and its corresponding analytical detection probability using normalized non-central/central approximation as a function of SNR for $N=10$ and $P_{\mathrm{fa}}=0.1$

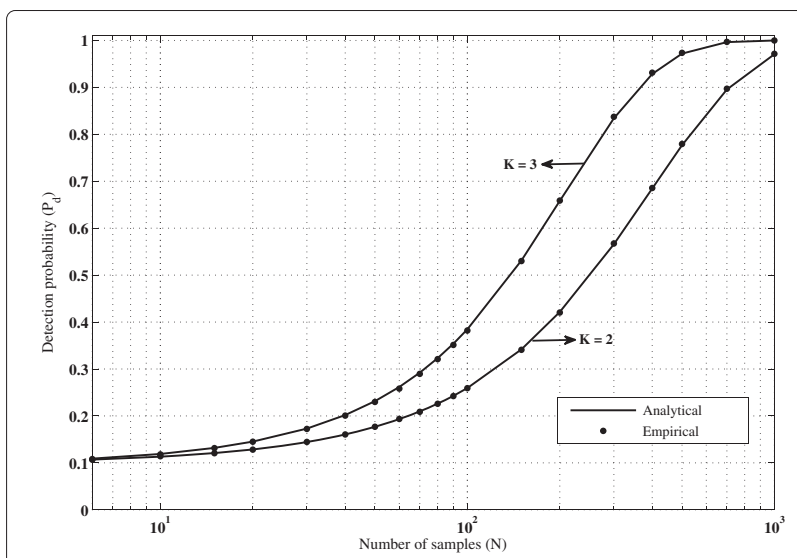

Fig. 7 Empirical detection probability of the SCN metric and its corresponding analytical detection probability using normalized non-central/central approximation as a function of the number of received samples for $\rho=-10 \mathrm{~dB}$, and $P_{\mathrm{fa}}=0.1$

$\left(P_{\mathrm{d}}<1\right)$, therefore, the approximation perfectly fits the empirical distribution. Thus, at an acceptable SNR value, the approximation does not show any degradation as $N$ and $K$ values change.

The performance comparison between the SCN detector, the LE detector, and the ED [5] is illustrated in Fig. 8. In this case, we set $K=3, N=500$, and $P_{\mathrm{fa}}=0.1$. If noise variance is perfectly known, results show that the LE detector outperforms the SCN detector as it is the optimal detector under this condition. However, noise variance is not known in practice. Accordingly, we considered a $0.3 \mathrm{~dB}$ noise uncertainty and the results are shown in Fig. 8. Results show that the SCN detector outperforms both the LE and ED as noise uncertainty is considered.

\section{Conclusions}

In this paper, we have considered the detection probability of the $\mathrm{SCN}$ detector. We have provided new statistics for the SCN metric, particularly for a $K$-dimensional cognitive radio system, by generalizing the form of the joint distribution of the ordered eigenvalues of central semicorrelated Wishart matrices. Then, we have considered a three-dimensional system and have derived the approximated detection probability by the use of normalized non-central/central approximation. The analytical results are validated through Monte Carlo simulations in different environments and with different system parameters. Results show that the approximation perfectly fits the empirical results at low SNR values; however, the approximation accuracy degrades as SNR increases. For a target false alarm probability, results prove that the probability of detection could be approximated using normalized noncentral/central approximation for all SNR values when the values of $N$ and $K$ are large enough. In addition, and by considering the noise uncertainty problem, results show 


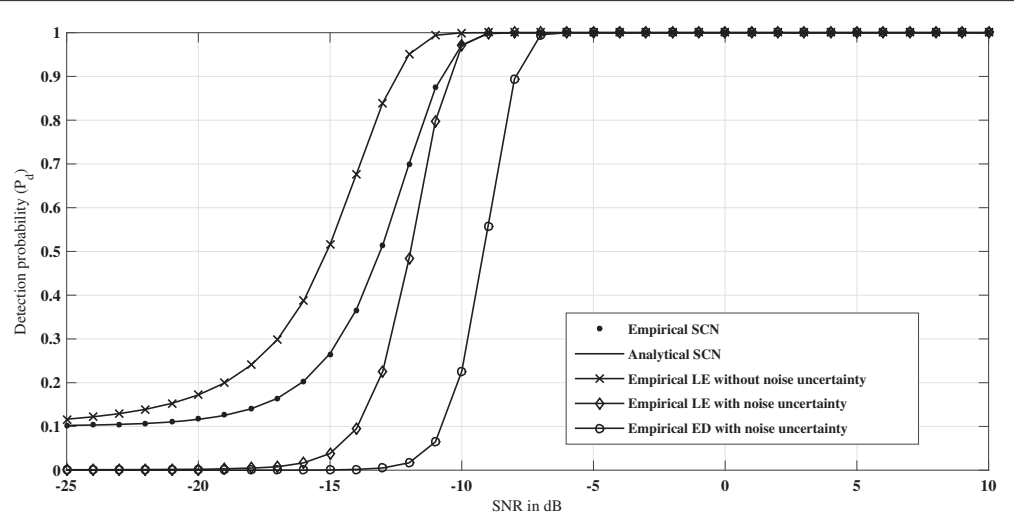

Fig. 8 Probability of detection of the SCN metric, LE, and energy detector with $0.3 \mathrm{~dB}$ noise uncertainty as a function of $S N R$ for $K=3, N=500$, and $P_{\mathrm{fa}}=0.1$

that the SCN detector outperforms the LE detector and the ED even with very small noise uncertainty value.

\section{Endnotes}

${ }^{1}$ The infinite sum of the hypergeometric function could be truncated to a finite sum with good accuracy if the $K, N$, and SNR are small. However, for a CR system, $N$ is usually large.

${ }^{2}$ The norm of the PU signal, $\|\boldsymbol{s}\|^{2}$, is still a random variable; however, this randomness decreases fast as $N$ increases and $\left(\|\boldsymbol{s}\|^{2} / N\right)$ can be well approximated by $\sigma_{s}^{2}$ for sufficient value of $\mathrm{N}$.

\section{Appendices}

\section{Appendix 1: proof of Theorem 1.}

Before we start the proof, it is required to give the following Lemma presenting the $n^{\text {th }}$ derivative of any function of the form $e^{-\frac{a}{x}}$ with respect to the variable $x$.

Lemma A.1. Let us define the function $f(x)=e^{-\frac{a}{x}}$, then the $n^{\text {th }}$ derivative of $f(x)$ is given by

$$
f^{(n)}(x)=\frac{a}{x^{2 n}} \cdot e^{-\frac{a}{x}} \cdot\left[\sum_{k=1}^{n}(-1)^{k+n} \mathcal{L}(n, k) x^{n-k} a^{k-1}\right]
$$

with $\mathcal{L}(n, k)$ the Lah number defined in (17).

Proof. It should be noted that, for any value of $n$, the $n^{\text {th }}$ derivative of $e^{-\frac{a}{x}}$ will result into the function $e^{-\frac{a}{x}}$ multiplied by sum of $(a / x)$ with different powers for $a$ and $x$. Regardless of the sign, it is found that the number multiplied by each component of this sum is exactly equals to the Lah number.

Following the pattern seen by calculating the derivative of $f(x)$ for small values of $n$, and matching the numbers at each derivative level, " $n$," with the Lah number $\mathcal{L}(n, k)$, it can be proved, by recurrence, that the $n^{\text {th }}$ derivative of the function $f(x)$ of the form $e^{-\frac{a}{x}}$ is given by (29).

Now, we proceed in proving Theorem 1. If we have $L$ equal eigenvalues $\left(\sigma_{p}=\cdots=\sigma_{q}\right)$, we must generalize the result of central semi-correlated Wishart with distinct correlation eigenvalues for arbitrary number of coincident eigenvalues by taking the limit of joint distribution given by Eq. 6 of [30] as follows:

$$
\begin{aligned}
& \lim _{\sigma_{p, q} \rightarrow \sigma_{p}} f(\lambda)=|\boldsymbol{\Phi}(\lambda)| \prod_{i=1}^{K} \xi\left(\lambda_{i}\right) \lim _{\sigma_{p, q} \rightarrow \sigma_{p}}(C \times|\mathbf{E}(\boldsymbol{\lambda}, \boldsymbol{\sigma})|) \\
& =|\boldsymbol{\Phi}(\boldsymbol{\lambda})| \prod_{i=1}^{K} \xi\left(\lambda_{i}\right) \lim _{\sigma_{p, q} \rightarrow \sigma_{p}}\left(\frac{\prod_{i<j}^{K} \sigma_{i} \sigma_{j}}{\prod_{i=1}^{K} \sigma_{i}^{N}(N-i) !} \cdot \frac{|\mathbf{E}(\boldsymbol{\lambda}, \boldsymbol{\sigma})|}{\prod_{i<j}^{K} \sigma_{j}-\sigma_{i}}\right)
\end{aligned}
$$

with $\sigma_{p, q}=\left[\sigma_{p}, \cdots, \sigma_{q}\right]$, and $C$ the normalization constant, $\boldsymbol{\Phi}(\lambda)$ is a Vandermonde matrix of entries $\lambda_{j}^{i-1}$, $\boldsymbol{E}(\boldsymbol{\lambda}, \boldsymbol{\sigma})$ is matrix of entries $e^{\frac{-\lambda_{j}}{\sigma_{i}}}$, and $\xi\left(\lambda_{i}\right)=\lambda_{i}^{N-K}$ [30, Table I]. To evaluate the limit in (30), we apply Lemma 2 from [39] to obtain

$$
\lim _{\sigma_{p, q} \rightarrow \sigma_{p}} \frac{\left|\begin{array}{cccc}
e\left(\lambda_{1}, \sigma_{1}\right) & \cdots & \cdots & e\left(\lambda_{K}, \sigma_{1}\right) \\
\vdots & \ddots & \ddots & \vdots \\
\prod_{i<j}\left(\lambda_{1}, \sigma_{p-1}\right) & \cdots & \cdots & e\left(\lambda_{K}, \sigma_{p-1}\right) \\
e^{(L-1)}\left(\lambda_{1}, \sigma_{p}\right) & \cdots & \cdots & e^{(L-1)}\left(\lambda_{K}, \sigma_{p}\right) \\
\vdots & \ddots & \ddots & \vdots \\
e^{(0)}\left(\lambda_{1}, \sigma_{p}\right) & \cdots & \cdots & e^{(0)}\left(\lambda_{K}, \sigma_{p}\right) \\
e\left(\lambda_{1}, \sigma_{q+1}\right) & \cdots & \cdots & e\left(\lambda_{K}, \sigma_{q+1}\right) \\
\vdots & \ddots & \ddots & \vdots \\
e\left(\lambda_{1}, \sigma_{K}\right) & \cdots & \cdots & e\left(\lambda_{K}, \sigma_{K}\right)
\end{array}\right|}{\prod_{\frac{i<j}{\sigma_{i} \neq \sigma_{j}}}^{K}\left(\sigma_{j}-\sigma_{i}\right) \cdot \Gamma_{L}(L)}
$$


with $e\left(\lambda_{i}, \sigma_{j}\right)=e^{-\frac{\lambda_{i}}{\sigma_{j}}}$, the required derivatives are evaluated using (29) and $\Gamma_{s}(l)=\prod_{i=1}^{s}(l-i)$ !. Then, the result is obtained by substituting (29) and (31) into (30) and simplifying.

In the case, we have several coincidences (e.g., $\sigma_{p, q}=\sigma_{p}$, $\sigma_{t, s}=\sigma_{s}$ ), the same analysis could be applied and the limit must be taken over all coincidence cases.

\section{Appendix 2: the CDF and PDF of SCN}

In the case where $\Omega_{3}$ is not a rank 1 matrix (we have more than one PU), then the eigenvalues of $\Sigma_{3}$ are not equal anymore. For sake of completeness of three-dimensional system case, we give in Theorem 3 and Corollary 2 the $\mathrm{CDF}$ and PDF of the SCN metric when all the correlation eigenvalues are distinct.

Theorem 3 (CDF of SCN of Central Semi-Correlated Wishart Matrix when $\Sigma_{3}$ has distinct eigenvalues). The $C D F$ of the $S C N$ of $3 \times 3$ central semi-correlated Wishart matrix $\mathbf{W} \sim \mathcal{C W}_{K}\left(N, \Sigma_{3}\right)$ when eigenvalues of $\Sigma_{3}$ are distinct is given by

$$
F_{1}(x)=C_{s} \cdot R_{3}(N, x), \quad z \geq 1,
$$

where $R_{3}(N, x)$ is defined in (33), $S_{3}(r, s, t, x)$ in (34), $\Delta_{3}(r, s, t, \mu, v, \varepsilon, x)$ in (35), and $C_{s}$ in (36).

$$
\begin{aligned}
& R_{i}(N, x)=S_{i}(N-2, N-1, N-3, x) \\
& -S_{i}(N-3, N-1, N-2, x) \\
& +S_{i}(N-3, N-2, N-1, x), i=3,4 \\
& S_{i}(r, s, t, x)=\Delta_{i}\left(r, s, t, \sigma_{2}^{-1}, \sigma_{1}^{-1}, \sigma_{3}^{-1}, x\right) \\
& -\Delta_{i}\left(r, s, t, \sigma_{3}^{-1}, \sigma_{1}^{-1}, \sigma_{2}^{-1}, x\right) \\
& -\Delta_{i}\left(r, s, t, \sigma_{1}^{-1}, \sigma_{2}^{-1}, \sigma_{3}^{-1}, x\right) \\
& +\Delta_{i}\left(r, s, t, \sigma_{3}^{-1}, \sigma_{2}^{-1}, \sigma_{1}^{-1}, x\right) \\
& +\Delta_{i}\left(r, s, t, \sigma_{1}^{-1}, \sigma_{3}^{-1}, \sigma_{2}^{-1}, x\right) \\
& -\Delta_{i}\left(r, s, t, \sigma_{2}^{-1}, \sigma_{3}^{-1}, \sigma_{1}^{-1}, x\right), i=3,4 \\
& \Delta_{3}(r, s, t, \mu, v, \varepsilon, x)=\left(\frac{r ! s !}{\mu^{r+1} \varepsilon^{s+1}}\right) \sum_{k=0}^{r} \sum_{u=0}^{s}\left[\frac{(k+u+t) !}{k ! u ! \mu^{-k} \varepsilon^{-u}} .\right. \\
& \times\left(\frac{1}{(\mu+\varepsilon+v)^{k+u+t+1}}\right. \\
& -\frac{x^{u}}{(\mu+\varepsilon x+v)^{k+u+t+1}} \\
& -\frac{x^{k}}{(\mu x+\varepsilon+v)^{k+u+t+1}} \\
& \left.\left.+\frac{x^{k+u}}{(\mu x+\varepsilon x+v)^{k+u+t+1}}\right)\right]
\end{aligned}
$$

$$
C_{s}=\frac{\left[\left(\sigma_{2}-\sigma_{1}\right)\left(\sigma_{3}-\sigma_{1}\right)\left(\sigma_{3}-\sigma_{2}\right)\right]^{-1}}{(N-1) !(N-2) !(N-3) ! \sigma_{1}^{N-2} \sigma_{2}^{N-2} \sigma_{3}^{N-2}}
$$

Proof. The proof could be summarized as follows:

1. Considering (18) and setting $K=3$.

2. Substituting the parameters from Theorem 1 with $L=1$.

3. Expanding the summation and using (19) with $p=q$.

4. Expanding the determinant and integrate using Eqs. (3.351.1) and (3.351.3) of [38].

Then, the result comes after simplification.

Corollary 2. The PDF of the SCN of $3 \times 3$ central semi-correlated Wishart matrix $\mathbf{W} \sim \mathcal{C} \mathcal{W}_{K}\left(N, \Sigma_{3}\right)$ when eigenvalues of $\Sigma_{3}$ are distinct is given by

$$
f_{1}(x)=C_{s} \cdot R_{4}(N, x), \quad z \geq 1,
$$

where $R_{4}(N, x)$ defined in (33), $S_{4}(r, s, t, x)$ in (34), $C_{s}$ in (36), and $\Delta_{4}(r, s, t, \mu, v, \varepsilon, x)$ in (38).

$$
\begin{aligned}
\Delta_{4}(r, s, t, \mu, v, \varepsilon, x)= & \left(\frac{r ! s !}{\mu^{r+1} \varepsilon^{s+1}}\right) \sum_{k=0}^{r} \sum_{u=0}^{s}\left[\left(\frac{(k+u+t) !}{k ! u ! \mu^{-k} \varepsilon^{-u}}\right) .\right. \\
& \times\left(\frac{x^{k+u-1}(v(k+u)-x(t+1)(\mu+\varepsilon))}{(\mu x+\varepsilon x+v)^{k+u+t+2}}\right. \\
& -\frac{x^{u-1}(u(\mu+v)-\varepsilon x(k+t+1))}{(\mu+\varepsilon x+v)^{k+u+t+2}} \\
& -\frac{x^{k-1}(k(\varepsilon+v)-\mu x(u+t+1))}{\left.\left.(\mu x+\varepsilon+v)^{k+u+t+2}\right)\right] .}
\end{aligned}
$$

Proof. Obtained by differentiating Eq. (32).

\section{Acknowledgements}

This work is supported by a program of cooperation between the Lebanese University and the Azem \& Saada social foundation (LU-AZM) and by CentraleSupélec (France).

\section{Competing interests}

The authors declare that they have no competing interests.

\section{Author details}

${ }^{1}$ SCEE/IETR, CentraleSupélec-Campus de Rennes, Rennes, France. ${ }^{2}$ Department of Physics and Electronics, Faculty of Sciences 1, Lebanese University, Hadath, Beirut, Lebanon. ${ }^{3}$ ECE Department, AUB, Bliss Street, Beirut, Lebanon.

Received: 29 September 2015 Accepted: 9 May 2016

Published online: 23 May 2016

\section{References}

1. J III Mitola, Cognitive radio: an integrated agent architecture for software defined radio, Computer Communication System Laboratory, Department of Teleinformatics, Royal Institute of Technology (KTH), (Stockholm, Sweden, 2000) 
2. Federal Communications Commission Spectrum Policy Task Force, Report of the spectrum efficiency working group. FCC (2002)

3. TYucek, H Arslan, A survey of spectrum sensing algorithms for cognitive radio applications. IEEE Commun. Surveys Tutorials. 11(1), 116-130 (2009). doi:10.1109/SURV.2009.090109

4. R Tandra, A Sahai, SNR walls for signal detection. IEEE J. Sel. Topics Signal Process. 2(1), 4-17 (2008). doi:10.1109/JSTSP.2007.914879

5. S Bahamou, A Nafkha, in EUSIPCO. Noise uncertainty analysis of energy detector: bounded and unbounded approximation relationship (IEEE, Morocco, 2013)

6. R Zhang, TJ Lim, Y-C Liang, Y Zeng, Multi-antenna based spectrum sensing for cognitive radios: a GLRT approach. IEEE Trans. Commun. 58(1), 84-88 (2010). doi:10.1109/TCOMM.2010.01.080158

7. A Taherpour, M Nasiri-kenari, S Gazor, Multiple antenna spectrum sensing in cognitive radios. IEEE Trans. Wirel. Commun. 9(2), 814-823 (2010). doi:10.1109/TWC.2009.02.090385

8. A Kortun, T Ratnarajah, M Sellathurai, C Zhong, CB Papadias, On the performance of eigenvalue-based cooperative spectrum sensing for cognitive radio. IEEE J. Sel. Topics Signal Process. 5(1), 49-55 (2011). doi:10.1109/JSTSP.2010.2066957

9. Y Zeng, Y-C Liang, Eigenvalue-based spectrum sensing algorithms for cognitive radio. IEEE Trans. Commun. 57(6), 1784-1793 (2009)

10. AM Tulino, S Verdu, Random matrix theory and wireless communications. (now Publishers Inc., USA, 2004)

11. Y Zeng, C Koh, Y-C Liang, in IEEE International Conference on Communication. Maximum eigenvalue detection: theory and application (IEEE, Beijing, 2008), pp. 4160-4164

12. P Bianchi, J Najim, G Alfano, M Debbah, in IEEE Information Theory Workshop. Asymptotics of eigenbased collaborative sensing, (2009), pp. 515-519. doi:10.1109/ITW.2009.5351479

13. P Bianchi, M Debbah, M Maida, J Najim, Performance of statistical tests for single-source detection using random matrix theory. IEEE Trans. Inf. Theory. 57(4), 2400-2419 (2011). doi:10.1109/TIT.2011.2111710

14. LS Cardoso, M Debbah, P Bianchi, J Najim, in Proc. IEEE International Symptoms Wireless Pervasive Computing. Cooperative spectrum sensing using random matrix theory (IEEE, Greece, 2008), pp. 334-338

15. F Penna, R Garello, M Spirito, Cooperative spectrum sensing based on the limiting eigenvalue ratio distribution in Wishart matrices. IEEE Commun. Lett. 13(7), 507-509 (2009)

16. F Penna, R Garello, D Figlioli, MA Spirito, in Proc. IEEE 4th International Conference CROWNCOM. Exact non-asymptotic threshold for eigenvalue-based spectrum sensing (IEEE, Germany, 2009), pp. 1-5

17. F Penna, R Garello, MA Spirito, in IEEE International Conference on Wireless and Mobile Computing, Networking and Communications. Probability of missed detection in eigenvalue ratio spectrum sensing, (2009), pp. 117-122. doi:10.1109/WiMob.2009.29

18. W Zhang, G Abreu, M Inamori, Y Sanada, Spectrum sensing algorithms via finite random matrices. IEEE Trans. Commun. 60(1), 164-175 (2012)

19. H Artes, D Seethaler, F Hlawatsch, Efficient detection algorithms for MIMO channels: a geometrical approach to approximate ML detection. IEEE Trans. Signal Process. 51(11), 2808-2820 (2003)

20. D Wubben, R Bohnke, V Kuhn, KD Kammeyer, in Proc. ITG Work. Smart Antennas. MMSE-based lattice-reduction for near-ML detection of MIMO systems (IEEE, Germany, 2004), pp. 106-113

21. J Maurer, G Matz, D Seethaler, in IEEE International Conference A.S.S.P 3. Low-Complexity and full-diversity MIMO detection based on condition number thresholding (IEEE, Honolulu, Hi, 2007), pp. 61-64

22. MD Batariere, TK Blankenship, JF Kepler, TP Krauss, I Lisica, S Mukthavaram, JW Porter, TA Thomas, FW Vook, in Proc. 55th IEEE Veh. Tech. Conf. (VTC), 1. Wideband MIMO mobile impulse response measurements at $3.7 \mathrm{GHz}$ (IEEE, Birmingham, AL, USA, 2002), pp. 26-30

23. V Erceg, P Soma, DS Baum, AJ Paulraj, in Proc. IEEE Int. Conf. Commun. (ICC), 1. Capacity obtained from multiple-input multiple-output channel measurements in fixed wireless environments at $2.5 \mathrm{GHz}$ (IEEE, New York, 2002), pp. 396-400

24. VA Marcenko, LA Pastur, Distribution of eigenvalues for some sets of random matrices. Math. USSR-Sbornik. 1(4), 457-483 (1967)

25. R Couillet, M Debbah, Random Matrix Theory for Wireless Communications. (Cambridge University Press, UK, 2011)

26. CA Tracy, H Widom, On orthogonal and symplectic matrix ensembles. Comm. Math. Phys. 177(3), 727-754 (1996)
27. M Johnstone, On the distribution of the largest eigenvalue in principal components analysis. Ann. Statist. 29, 295-327 (2001)

28. JH Curtiss, On the distribution of the quotient of two chance variables. Ann. Math. Statist. 12(4), 409-421 (1941). doi:10.1214/aoms/1177731679

29. ON Feldheim, S Sodin, A universality result for the smallest eigenvalues of certain sample covariance matrices. Geometric and Funct. Anal. 20(1), 88-123 (2010). doi:10.1007/s00039-010-0055-x

30. M Matthaiou, MR McKay, PJ Smith, JA Nossek, On the condition number distribution of complex wishart matrices. IEEE Trans. Commun. 58(6), 1705-1717 (2010)

31. H Kobeissi, Y Nasser, O Bazzi, Y Louet, A Nafkha, in XXXIth URSI General Assembly and Scientific Symposium (URSI GASS). On the performance evaluation of eigenvalue-based spectrum sensing detector for MIMO systems, (2014), pp. 1-4. doi:10.1109/URSIGASS.2014.6929235

32. L Wei, O Tirkkonen, in IEEE 20th International Symposium on Personal, Indoor and Mobile Radio Communications. Cooperative spectrum sensing of OFDM signals using largest eigenvalue distributions, (2009), pp. 2295-2299. doi:10.1109/PIMRC.2009.5449798

33. MK Simon, The Nuttall Q function - its relation to the Marcum Q function and its application in digital communication performance evaluation. IEEE Trans. Commun. 50(11), 1712-1715 (2002). doi:10.1109/TCOMM.2002.805270

34. M Matthaiou, DI Laurenson, C-X Wang, On analytical derivations of the condition number distributions of dual non-central Wishart matrices. IEEE Trans. Wirel. Commun. 8(3), 1212-1217 (2009)

35. WY Tan, RP Gupta, On approximating the non-central Wishart distribution with Wishart distribution. Commun. Stat. Theory Method. 12(22), 2589-2600 (1983)

36. S Jin, MR McKay, X Gao, I Collings, MIMO multichannel beamforming: SER and outage using new eigenvalue distributions of complex noncentral Wishart matrices. IEEE Trans. Commun. 56(3), 424-434 (2008)

37. XW Cui, QT Zhang, ZM Feng, Generic procedure for tightly bounding the capacity of MIMO correlated Rician fading channels. IEEE Transactions on Communication. 53(5), 890-898 (2005)

38. IS Gradshteyn, IM Ryzhik, Table of Integrals, Series, and Products, 7th edn. (ELSEVIER, USA, 2007)

39. M Chiani, MZ Win, H Shin, in Proc. IEEE GLOBECOM. Capacity of MIMO systems in the presence of interference (IEEE, San Francisco, 2006), pp. 1-6

\section{Submit your manuscript to a SpringerOpen ${ }^{\circ}$ journal and benefit from:}

- Convenient online submission

- Rigorous peer review

- Immediate publication on acceptance

- Open access: articles freely available online

- High visibility within the field

- Retaining the copyright to your article

Submit your next manuscript at springeropen.com 